\title{
Does dexamethasone inhibit anticancer activity of cetuximab in squamous cell carcinoma cell lines of the head and neck?
}

\author{
JENS WAGENBLAST ${ }^{1}$, MEHRAN BAGHI ${ }^{1}$, SUSANNE MÖRTEL ${ }^{1}$, DANIEL HIRTH ${ }^{1}$, LAURA THRON $^{1}$, \\ CHRISTOPH ARNOLDNER ${ }^{2}$, WOLFGANG GSTÖTTNER ${ }^{2}$, ANGELIKA MAY ${ }^{1}$ and MARKUS HAMBEK ${ }^{1}$ \\ ${ }^{1}$ ENT Department, Goethe University Medical School, Frankfurt am Main, Germany; \\ ${ }^{2}$ ENT Department, Medical University of Vienna, Austria
}

Received February 23, 2009; Accepted April 27, 2009

DOI: 10.3892/or_00000421

\begin{abstract}
Glucocorticoids such as dexamethasone are widely used as comedication in the treatment of head and neck cancer, e.g., to improve appetite and decrease weight loss and fatigue in patients with advanced disease or as antiallergic and antiemetic prophylaxis during anti-EGFR therapy. However, the literature suggests that dexamethasone induces resistance to antineoplastic agents in many solid tumor models in vitro and in vivo. Since this phenomenon has never been investigated in head and neck cancer, the present study was conducted to investigate the effect of dexamethasone on the antiproliferative activity of cetuximab in vitro in squamous cell carcinoma of the head and neck (SCCHN) cell lines. The antiproliferative effect of the anti-EGFR agent cetuximab alone and in combination with increasing concentrations of dexamethasone was examined in eight SCCHN cell lines at three different time-points (24, 48 and $72 \mathrm{~h}$ ). Cell growth inhibition and viability were measured quantitatively using WST and LDH assays. Absolute tumor cell numbers were determined by cell counting in a Rosenthal chamber. Cetuximab alone inhibited the growth of all eight SCCHN cell lines significantly $(\mathrm{p}=0.008)$. In some cases the addition of dexamethasone reduced the antiproliferative activity of cetuximab $(p \leq 0.038)$ but remained significant in all of the eight SCCHN cell lines compared with untreated controls $(\mathrm{p} \leq 0.028)$ at each drug concentration and each time-point. In contrast to the results reported for other tumor models, in our study dexamethasone showed in the majority of the evaluated dexamethasone drug concentrations and timepoints no inhibition of the cytotoxic activity of cetuximab. The reasons for these discrepant findings are unclear but may be related to the degree of tumor cell differentiation or
\end{abstract}

Correspondence to: Dr Jens Wagenblast, ENT Department, University Hospital, Theodor-Stern-Kai 7, D-60590 Frankfurt/ Main, Germany

E-mail: jens.wagenblast@kgu.de

Key words: cetuximab, dexamethasone, squamous cell carcinoma of the head and neck cell lines, resistance, apoptosis proliferation rate. Thus, further studies are required to elucidate the molecular mechanisms underlying the interaction between dexamethasone and cetuximab in different tumors.

\section{Introduction}

Cancer of the head and neck most frequently affects the oral cavity, pharynx or larynx and accounts for more than $5 \%$ of all malignancies worldwide. In 2002, more than 500,000 new cancer diagnoses and more than 300,000 deaths were attributable to this disease (1). Most head and neck cancers are squamous cell carcinomas (SCCHN). The prognosis depends primarily on disease stage and performance status at the time of diagnosis $(2,3)$. Treatment options are limited for metastatic and/or recurrent disease, and there is an urgent need for new, well tolerated therapies (4). Molecular targeted therapies, especially those targeting the epidermal growth factor receptor (EGFR) have recently attracted attention as promising candidates for the treatment of head and neck cancers (5). EGFR overexpression in head and neck cancer has been correlated with a poor prognosis $(6,7)$, and the potential therapeutic value of modulating the EGFR signalling pathway is reflected by the broad range of molecular EGFR inhibitors developed in recent years. Cetuximab is an immunoglobuline G1 monoclonal antibody with a higher affinity to the extracellular domain of the receptor compared with its natural ligands. The drug is under active investigation as a promising anticancer agent in general (8) and especially in SCCHN (9). A significant activity has been demonstrated in clinical trials of EGFR-blocking antibodies (10). Skin toxicity is the most important side effect of treatment with EGFR inhibitors including cetuximab. Severe infusion reactions have also been reported in rare cases and described as hypersensitivity (10), anaphylactic (11) or allergic reactions (12), although the mechanism of this adverse effect has not yet been identified. In most cases the cetuximab infusion was stopped after an infusion reaction occurred. Melichar et al (13), however, reported the successful continuation of cetuximab infusion after an infusion reaction when glucocorticoids were added and the patients were monitored in an intensive care unit. Apart from antiallergic prophylaxis during anti-EGFR therapy, glucocorticoids such as dexamethasone are widely used as a comedication in the treatment of head and neck cancer, e.g., to improve appetite or decrease weight 
A

Cetuximab mono and in combination with dexamethasone versus untreated control (24h)

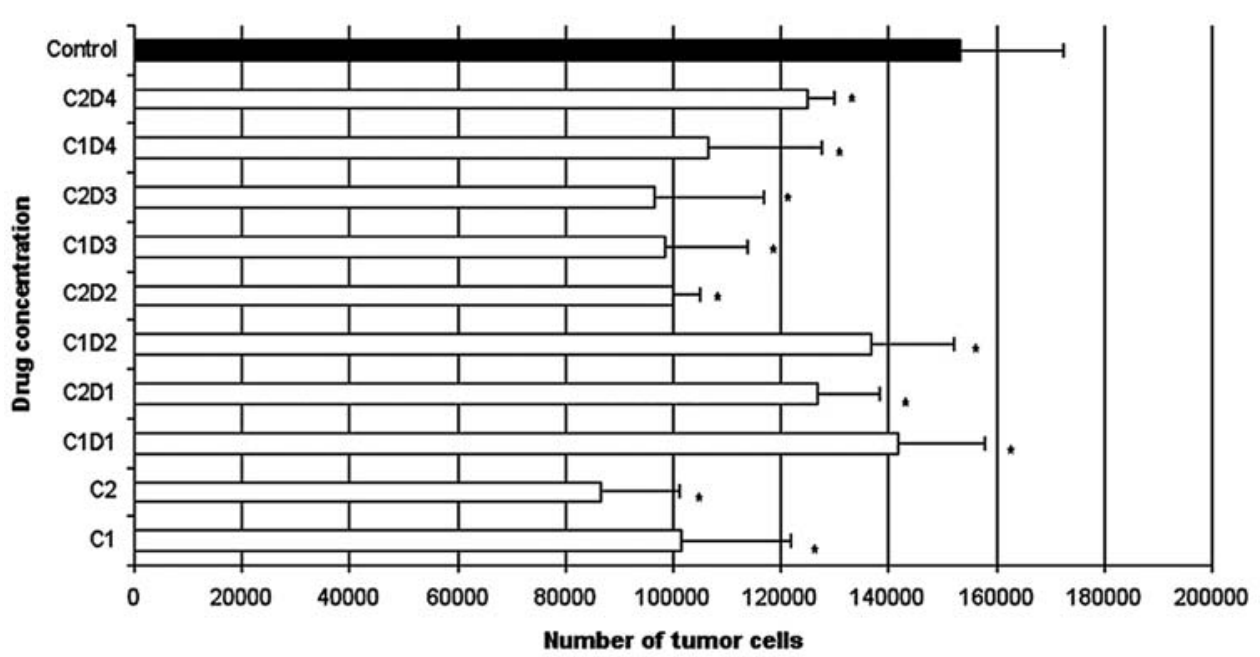

B

Cetuximab mono and in combination with dexamethasone versus untreated control (48h)

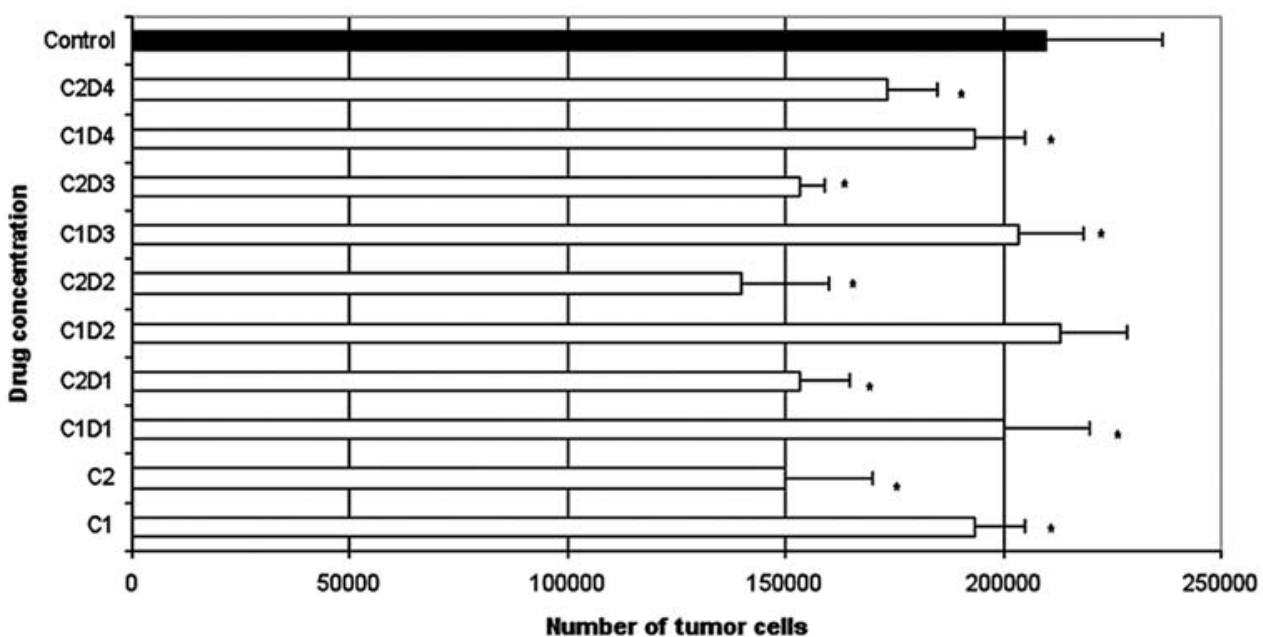

C

Cetuximab mono and in combination with dexamethasone versus untreated control (72h)

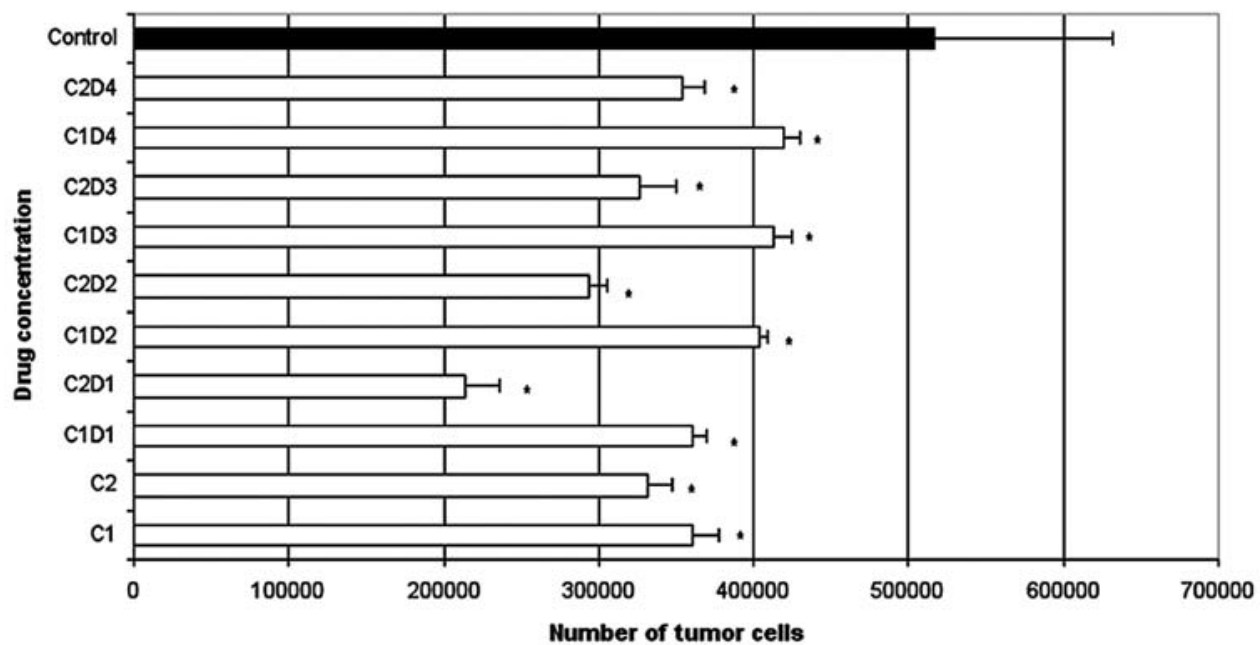

Figure 1. (A-C) The growth-inhibitory effect of cetuximab at two concentrations $(\mathrm{C} 1=0.5$ and $\mathrm{C} 2=5.0 \mu \mathrm{mol} / \mathrm{l})$, administered either alone or in combination with one of four concentrations of dexamethasone (D1=1.0 $\mu \mathrm{mol} / 1, \mathrm{D} 2=2.5 \mu \mathrm{mol} / 1, \mathrm{D} 3=5.0 \mu \mathrm{mol} / 1, \mathrm{D} 4=10.0 \mu \mathrm{mol} / 1)$ in the SCCHN cell line PJ-34 which was representative for the eight tumor cell lines investigated (yellow columns). The corresponding untreated tumor cell line (black column) served as a control and was incubated only with the cell-type specific medium Quantum 263 with L-glutamine. The absolute tumor cell numbers in treated and control cell lines were determined in a Rosenthal chamber at 24, 48 and $72 \mathrm{~h}$ after treatment or incubation with Quantum 263 (controls), respectively. Mean values of three independent experiments with standard deviation are shown. Similar results were obtained in all eight tumor cell lines investigated. Compared with the control group, single agent cetuximab had a highly significant $(\mathrm{p}=0.008)$ antiproliferative effect at both concentrations $(0.5$ and $5.0 \mu \mathrm{mol} / \mathrm{l})$ and at all time-points $(24$, 48 and $72 \mathrm{~h}$ ) in all eight squamous cell carcinoma cell lines. Cetuximab was also found to be significantly active at both concentrations and at every time-point when it was given in combination with dexamethasone at all concentrations compared with untreated controls using the Wilcoxon test for matched pairs samples $(\mathrm{p} \leq 0.021$ at $24 \mathrm{~h}, \mathrm{p} \leq 0.028$ at $48 \mathrm{~h}$ and $\mathrm{p} \leq 0.008$ at $72 \mathrm{~h}$ ). 
loss, fatigue or nausea in patients with advanced disease. However, in the past few years several publications have discussed the potential of glucocorticoids like dexamethasone to induce resistance to antineoplastic agents $(14,15)$. Thus, the question arises if dexamethasone used to prevent or treat allergic reactions to cetuximab may also lead to resistance to the agent in the treatment of SCCHN. The primary objective of our study was to investigate the interaction of dexamethasone and cetuximab in several SCCHN cell lines.

\section{Materials and methods}

Eight different SCCHN cell lines were used in this study. PE/CA-PJ-15, PE/CA-PJ-34, PE/CA-PJ-41 and PE/CA-PJ-49 cells were obtained from ECACC (European Collection of Cell Cultures, Salisbury, Wiltshire, UK), and Cal-27 and Kyse-140 cells were purchased from DSMZ GmbH (Braunschweig, Germany). CLS-354 and UM-SCC-14C were obtained from CLS Cell Line Service (Eppelheim, Germany). The fibroblast cell line was a gift of the Department of Dermatology, University Hospital, Frankfurt/Main, Germany. Cetuximab was obtained from Merck (Darmstadt, Germany) and dexamethasone from Sigma-Aldrich (Munich, Germany). The cell lines were cultivated according to the instructions of the suppliers without antibiotics at $37^{\circ} \mathrm{C}$ in the cell-type specific medium Quantum 263 with L-glutamine (PAA Laboratories GmbH, Pasching, Austria). Cells were seeded in 96-multiwell plates $(1 \times 100,000$ cells/well), and after incubation for $24 \mathrm{~h}$, the cells were treated with cetuximab alone or in combination with dexamethasone for 24,48 or $76 \mathrm{~h}$, respectively. In all experiments described in this publication, cetuximab was used in two different concentrations $(0.5$ and $5.0 \mu \mathrm{mol} / \mathrm{l})$, while dexamethasone was used at four increasing concentrations ranging from 1 to $10 \mu \mathrm{mol} / \mathrm{l}$; these concentrations are comparable with the clinically achievable tissue concentrations of the drug (16). The number of cells was determined in a Rosenthal chamber after 24,48 and $72 \mathrm{~h}$ of treatment. Cell viability and cell killing were determined by a WST and lactate dehydrogenase (LDH) assay, respectively. For the WST assay, $1 \times 10^{5}$ cells per well were cultivated in a 96-well plate for $24 \mathrm{~h}$ and then treated with the aforementioned concentrations of cetuximab and dexamethasone for 24,48 or $72 \mathrm{~h}$, respectively. WST $(10 \mu \mathrm{l})$ at $5 \mathrm{~g} / \mathrm{l}$ (Roche Diagnostics, Mannheim, Germany) were added to the medium in triplicate at each dose and incubated for $1 \mathrm{~h}$ at $37^{\circ} \mathrm{C}$. Absorbance was measured at $450 \mathrm{~nm}$ using a microplate reader. LDH activity in the culture medium was measured with the Cytotoxicity Detection Kit plus purchased from Roche Mannheim, Germany. Briefly, cells were incubated in a 96-well microplate (Falcon, Franklin Lakes, NJ, USA), with 5,000 cells in $200 \mu 1$ seeded per well with Quantum 263 PAA. After 24 h, the medium was removed and replaced either by the same medium containing cetuximab with or without dexamethasone at the concentrations specified above or drug-free medium (low controls), or medium containing 1\% Triton X-100 (Sigma Chemical Co.) to determine total cellular LDH (high controls). After 24, 48 or $72 \mathrm{~h}$ of treatment, $100-\mu 1$ samples were removed from the wells and transferred to another well-plate, $100 \mu 1$ of the $\mathrm{LDH}$ assay reaction mixture were added to each well, and cells were subsequently incubated for $30 \mathrm{~min}$ at room temperature. During the incubation period, the micro-plates were protected from light. The optical density of each well was determined using a microplate reader (Dynatech Laboratories, Chantilly, VA, USA) at a wavelength of $490 \mathrm{~nm}$ with a reference wavelength of $630 \mathrm{~nm}$. Each experiment was done in triplicate. For statistical analysis, a Wilcoxon test for matched pairs (dependent samples) was performed using SPSS 13.0 software for Windows.

\section{Results}

Cetuximab alone showed a highly significant $(p=0.008)$ antiproliferative effect compared with the control group at both concentrations $(0.5$ and $5.0 \mu \mathrm{mol} / \mathrm{l})$ and at all timepoints $(24,48$ and $72 \mathrm{~h})$ in all eight cell lines.

Since the present study was conducted to investigate the effect of dexamethasone on the antiproliferative activity of cetuximab in vitro in squamous cell carcinoma of the head and neck (SCCHN) cell lines, cetuximab was combined with four different concentrations of dexamethasone.

When cetuximab was given in combination with dexamethasone at four different concentrations, the drug also exerted significant antiproliferative activity compared with untreated controls at both concentrations and each time-point considered $(\mathrm{p} \leq 0.021$ at $24 \mathrm{~h}, \mathrm{p} \leq 0.028$ at $48 \mathrm{~h}$ and $\mathrm{p} \leq 0.008$ at $72 \mathrm{~h})$, although there was a significantly $(\mathrm{p}<0.05)$ higher tumor cell number in particular cases of combination treatment compared with cetuximab monotherapy.

Fig. 1 show the growth-inhibitory effect of cetuximab at two concentrations $(\mathrm{C} 1=0.5$ and $\mathrm{C} 2=5.0 \mu \mathrm{mol} / \mathrm{l})$, administered either alone or in combination with one of four concentrations of dexamethasone (D1=1.0 $\mu \mathrm{mol} / 1, \mathrm{D} 2=2.5 \mu \mathrm{mol} / \mathrm{l}$, $\mathrm{D} 3=5.0 \mu \mathrm{mol} / 1, \mathrm{D} 4=10.0 \mu \mathrm{mol} / \mathrm{l})$ in the $\mathrm{SCCHN}$ cell line PJ-34 which was representative for the eight tumor cell lines investigated. Similar results were obtained in all eight tumor cell lines investigated. Tumor cell viability and tumor cell decline were determined by the WST and LDH assay in each cell line and at each drug concentration of cetuximab alone or in combination with dexamethasone. The growth inhibition and tumor cell decline shown in Fig. 2 for PJ-34 cells (24-72 h) are representative for the cell lines investigated.

\section{Discussion}

For many years glucocorticoids have been used uncritically as comedication in patients with advanced head and neck cancer, e.g., to increase appetite, reduce weight loss and fatigue, or prevent nausea and allergic reactions. It was not until Herr and Pfitzenmaier (17) hypothesized an inhibition of the antineoplastic effect of various anticancer agents by glucocorticoids that this potential adverse interaction gained attention in the medical literature, both with regard to its importance in daily oncology practice and for patient outcome.

Cetuximab has proven to be a highly effective anticancer drug in several clinical trials. It was shown to prolong survival in patients with recurrent and/or metastatic SCCHN when given as primary therapy or after failure of platinum-based chemotherapy compared with various second-line therapies. 


\section{Results of the WST assay in the PJ-34 cell line (24 to $72 \mathrm{~h}$ ). \\ WST in PJ 34 tumor cells (24-72h)}

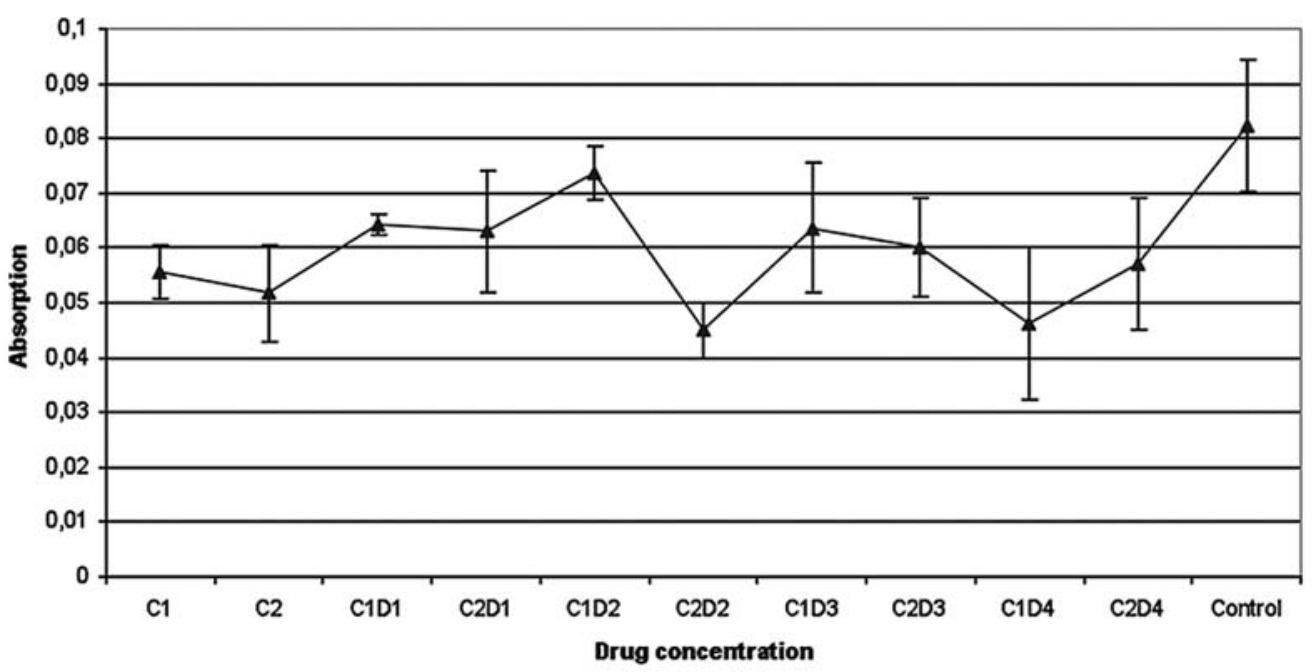

B

Results of the LDH assay in the PJ34 cell line (24 to $72 \mathrm{~h}$ ).

LDH Absorption in PJ 34 tumor cells (24-72h)

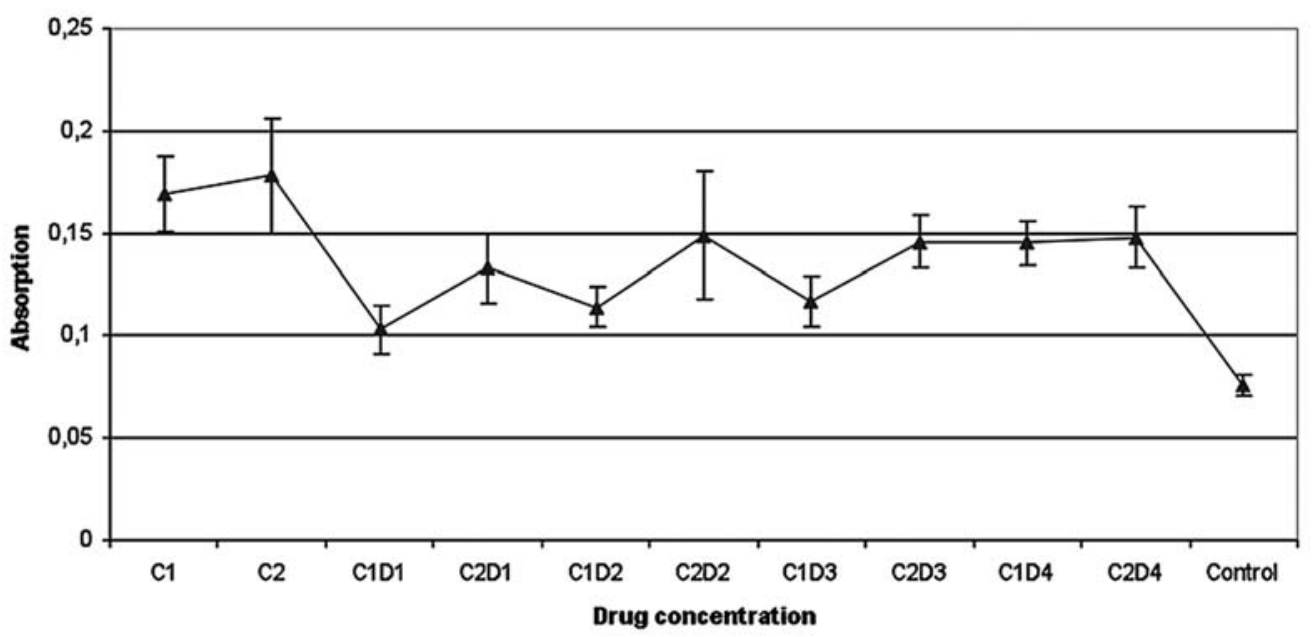

Figure 2. Tumor cell viability and tumor cell decline were determined by the WST and LDH assay in each cell line and at each drug concentration of cetuximab alone or in combination with dexamethasone. The growth inhibition and tumor cell decline shown in (A) and (B) for PJ-34 cells (24-72 h) are representative for the cell lines investigated. Mean values of three independent experiments with standard deviation are shown. Similar results were obtained for all eight cell lines investigated.

Remarkably, the addition of cetuximab did not increase the toxicity of chemotherapy $(10,18)$. In several treatment protocols containing cetuximab or other anti-EGFR therapies, comedication with dexamethasone is given because of its antiallergic and antiemetic effect. Against this background, it is important to know if glucocorticoids such as dexamethasone may inhibit the antineoplastic effect of cetuximab in the treatment of head and neck cancer. To our knowledge, the present in vitro study was the first to address this question, and it is reassuring that our results showed, in the majority of dexamethasone drug concentrations and time-points studied, no inhibition of the cytotoxic activity of cetuximab suggesting no evidence for an adverse interaction between cetuximab and dexamethasone in head and neck cancer cell lines. So these findings are partially in contrast to those reported by Zhang et al (19) who demonstrated glucocorticoid-induced resistance to chemotherapy in the majority of cell lines derived from various malignancies including brain, breast, cervix, melanoma and neuroblastoma. Gassler et al (14) found an anti-apoptotic effect of glucocorticoids in tissue samples from lung cancer. Glucocorticoids are also highly likely to induce resistance and accelerated tumor growth in patients with prostate, renal cell, bladder and testicular cancer (20). The reasons for our discrepant findings remain unclear although intrinsic properties of tumor cells may play a role, suggesting a more cell type-specific effect of glucocorticoids. This behavior would be in line with the complexity of tumor cell biology, as indicated by the well established pro-apoptotic and antiproliferative effect of glucocorticoids in lymphoid cells, as demonstrated by other research groups $(21,22)$. The mechanisms by which glucocorticoids induce apoptosis in lymphoid cells are well studied and include, among others, 
depolarization of the mitochondrial membrane and enhanced expression of the death receptor CD95 and its ligand, followed by activation of the caspase pathway (23). Those mechanisms underlying the glucocorticoid action in lymphoid cells were shown to be blocked in established human lung and breast cancer cell lines, thereby inhibiting chemotherapy and radiation-induced apoptosis. It remains unclear, however, how glucocorticoids mediate these cell type-specific effects that are known to be related to a functional glucocorticoid receptor $(22,24)$. Furthermore, there is convincing evidence for some unfavourable clinical effects of systemic glucocorticoids, e.g., an increased metastatic potential in breast cancer patients and an enhanced risk of skin cancer and lymphomas (25). Many studies in animal models have shown that administration of dexamethasone results in immunosuppression which may exacerbate metastatic spread and accelerate tumor growth (26). This could explain why glucocorticoids inhibited the effect of conventional chemotherapy with cisplatin and 5 -fluorouracil in some solid tumor models, while they were shown to be beneficial as an adjunct to bortezomib in the treatment of hematologic malignancies (27). Considering our results, the data obtained with glucocorticoids in cancers of the prostate, bladder, kidney, bone, brain, breast and cervix, as well as in melanoma and neuroblastoma can not be applied to solid tumors in general. One explanation for the divergent effects of dexamethasone in solid tumor models may be the cell type specific expression of glucocorticoid receptor coactivators and corepressors, as has been proposed to explain the opposite effects of tamoxifen on mammary versus endometrial tissues (28). Further data strongly suggest that tissue-specific differences in dexamethasone-induced apoptosis versus survival outcomes may be due to cell typespecific transcriptional regulation.

Finally, our findings that dexamethasone had in the majority of dexamethasone concentrations and time-points considered no severe negative effect on cetuximab activity in the eight different SCCHN cell lines investigated in vitro, could be explained by cetuximab signal transduction itself. Actually, it cannot be excluded that both the pro-apoptotic and anti-apoptotic effects of dexamethasone in solid tumor models are related to an interaction of the glucocorticoid with the signal transduction of antineoplastic agents. In our opinion it seems possible that complex resistance mechanisms, cell differentiation features and proliferation properties of different tumor cell lines influence the effects of dexamethasone as a result of subspecialisation of cancer cells in these models. Considering the contrasting findings of our study and those reported in the literature, it appears reasonable to suggest that the effect of dexamethasone varies both with the type of antineoplastic agent and the type of cancer.

Considering our results, the data obtained with glucocorticoids in cancers of solid tumors such as prostate, bladder, kidney, bone, brain, breast and cervix, as well as in melanoma and neuroblastoma by others, can not be applied to solid tumors in general. One explanation for the divergent effects of dexamethasone in solid tumor models may be the cell type-specific expression of glucocorticoid receptor coactivators and corepressors.

Further in vivo investigations are planned to substantiate our findings in squamous cell carcinoma cell lines of the head and neck to proof the clinical impact of dexamethasone on cetuximab treatment in this tumor entity.

\section{Acknowledgements}

We thank Erika Weith, Helena Baumann and Jeanne DufrasneMelchers for their excellent technical assistance.

\section{References}

1. Kamangar F, Dores GM and Anderson WF: Patterns of cancer incidence, mortality, and prevalence across five continents: defining priorities to reduce cancer disparities in different geographic regions of the world. J Clin Oncol 24: 2137-2150, 2006.

2. Waldfahrer F, Maldea L, Altendorf-Hofmann A and Iro H: Evaluation of the 'new' TNM classification of head and neck tumors in 3247 patients. HNO 47: 957-962, 1999.

3. Kowalski LP and Carvalho AL: Natural history of untreated head and neck cancer. Eur J Cancer 36: 1032-1037, 2000.

4. Wagenblast J, Hambek M, Baghi M, et al: Antiproliferative activity of bortezomib alone and in combination with cisplatin or docetaxel in head and neck squamous cell carcinoma cell lines. J Cancer Res Clin Oncol 134: 323-330, 2008.

5. Arteaga C: Targeting HER1/EGFR: a molecular approach to cancer therapy. Semin Oncol 30 (Suppl. 7): S3-S14, 2003.

6. Herbst RS and Langer CJ: Epidermal growth factor receptors as a target for cancer treatment: the emerging role of IMC-C225 in the treatment of lung and head and neck cancers. Semin Oncol 29 (Suppl. 4): S27-S36, 2002.

7. Ang KK, Berkey BA, Tu X, et al: Impact of epidermal growth factor receptor expression on survival and pattern of relapse in patients with advanced head and neck carcinoma. Cancer Res 62: 7350-7356, 2002.

8. Huang S, Armstrong EA, Benavente S, Chinnaiyan P and Harari PM: Dual-agent molecular targeting of the epidermal growth factor receptor (EGFR): combining anti-EGFR antibody with tyrosine kinase inhibitor. Cancer Res 64: 5355-5362, 2004.

9. Vermorken JB, Trigo J, Hitt R, et al: Open-label, uncontrolled, multicenter phase II study to evaluate the efficacy and toxicity of cetuximab as a single agent in patients with recurrent and/or metastatic squamous cell carcinoma of the head and neck who failed to respond to platinum-based therapy. J Clin Oncol 25: 2171-2177, 2007

10. Bonner JA, Harari PM, Giralt J, et al: Radiotherapy plus cetuximab for squamous-cell carcinoma of the head and neck. $\mathrm{N}$ Engl J Med 354: 567-578, 2006.

11. Cunningham D, Humblet Y, Siena S, et al: Cetuximab monotherapy and cetuximab plus irinotecan in irinotecan-refractory metastatic colorectal cancer. N Engl J Med 351: 337-345, 2004.

12. Saltz LB, Meropol NJ, Loehrer PJ Sr, Needle MN, Kopit J and Mayer RJ: Phase II trial of cetuximab in patients with refractory colorectal cancer that expresses the epidermal growth factor receptor. J Clin Oncol 22: 1201-1208, 2004.

13. Melichar B, Cerman J Jr and Malirova E: Successful management of infusion reaction accompanying the start of cetuximab therapy. Support Care Cancer 15: 445-449, 2007.

14. Gassler N, Zhang C, Wenger T, et al: Dexamethasone-induced cisplatin and gemcitabine resistance in lung carcinoma samples treated ex vivo. Br J Cancer 92: 1084-1088, 2005.

15. Zhang C, Kolb A, Mattern J, et al: Dexamethasone desensitizes hepatocellular and colorectal tumours toward cytotoxic therapy. Cancer Lett 242: 104-111, 2006.

16. Brady ME, Sartiano GP, Rosenblum SL, Zaglama NE and Bauguess CT: The pharmacokinetics of single high doses of dexamethasone in cancer patients. Eur J Clin Pharmacol 32: 593-596, 1987.

17. Herr I and Pfitzenmaier J: Glucocorticoid use in prostate cancer and other solid tumours: implications for effectiveness of cytotoxic treatment and metastases. Lancet Oncol 7: 425-430, 2006.

18. Vermorken JB, Herbst RS, Leon X, Amellal N and Baselga J: Overview of the efficacy of cetuximab in recurrent and/or metastatic squamous cell carcinoma of the head and neck in patients who previously failed platinum-based therapies. Cancer 112: 2710-2719, 2008. 
19. Zhang C, Beckermann B, Kallifatidis G, et al: Corticosteroids induce chemotherapy resistance in the majority of tumour cells from bone, brain, breast, cervix, melanoma and neuroblastoma. Int J Oncol 29: 1295-1301, 2006.

20. Zhang C, Mattern J, Haferkamp A, et al: Corticosteroid-induced chemotherapy resistance in urological cancers. Cancer Biol Ther 5: 59-64, 2006.

21. Rutz HP and Herr I: Interference of glucocorticoids with apoptosis signaling and host-tumor interactions. Cancer Biol Ther 3: 715-718, 2004.

22. Herr I, Ucur E, Herzer K, et al: Glucocorticoid cotreatment induces apoptosis resistance toward cancer therapy in carcinomas. Cancer Res 63: 3112-3120, 2003.

23. Planey SL and Litwack G: Glucocorticoid-induced apoptosis in lymphocytes. Biochem Biophys Res Commun 279: 307-312, 2000 .

24. Wu W, Chaudhuri S, Brickley DR, Pang D, Karrison T and Conzen SD: Microarray analysis reveals glucocorticoid-regulated survival genes that are associated with inhibition of apoptosis in breast epithelial cells. Cancer Res 64: 1757-1764, 2004.
25. Iversen HG and Hjort GH: The influence of corticoid steroids on the frequency of spleen metastases in patients with breast cancer. Acta Pathol Microbiol Scand 44: 205-212, 1958.

26. Fidler IJ, Gersten DM and Kripke ML: Influence of immune status on the metastasis of three murine fibrosarcomas of different immunogenicities. Cancer Res 39: 3816-3821, 1979.

27. Jagannath S, Richardson PG, Barlogie B, et al: Bortezomib in combination with dexamethasone for the treatment of patients with relapsed and/or refractory multiple myeloma with less than optimal response to bortezomib alone. Haematologica 91: 929-934, 2006.

28. Shang Y and Brown M: Molecular determinants for the tissue specificity of SERMs. Science 295: 2465-2468, 2002. 